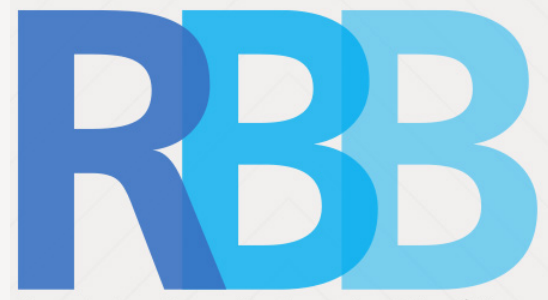

Revista Brasileira de Bioética

George Felipe de Moura Batista Curso de Medicina da Universidade Federal do Rio Grande do Norte. Natal, RN, Brasil

georgefelipe1997@gmail.com

Gustavo da Cunha Lima Freire Departamento de Morfologia da Universidade Federal do Rio Grande do Norte. Natal, RN, Brasil gustavo@cb.ufrn.com

\section{Análise do ensino da morte e do morrer na graduação médica brasileira}

\section{Analysis of the teaching of death and dying in the Brazilian bachelor in medicine}

Resumo: : No Brasil, as concepções sobre morte modificaramse ao longo da história, inclusive no processo de formação médica. Nos últimos anos, o ato de morrer aumentou nos leitos hospitalares, cabendo ao médico à função de enfrentá-lo a qualquer custo. Desse modo, tendo em vista os atuais dilemas éticos vivenciados por esses profissionais no processo de morte, é importante a análise e reflexão acerca do tema. Com base nisso, o objetivo desta pesquisa foi observar os tópicos relacionados à morte que constam no currículo médico das universidades brasileiras. Trata-se de um estudo documental qualitativo e descritivo, executado por meio da observação da ementa, currículo e proposta pedagógica dos 50 melhores cursos de medicina do país, conforme o Ranking Universitário Folha. O trabalho possibilitou evidenciar o déficit na abordagem da tanatologia nesses cursos, ressaltando a necessidade de modificação dessa realidade ainda no período acadêmico, tanto pela estruturação de ligas acadêmicas, quanto pelo ensino continuado do conteúdo e capacitação do corpo docente.

Palavras-chave: Morte, universidades, Brasil, educação médica, tanatologia.

Abstract: In Brazil, conceptions about death have changed throughout history, also in the medical training process. In the last years, the act of dying increased in hospital beds, and the physician has the function of confronting it at any cost. Thus, in view of the current ethical dilemmas experienced by these professionals in the death process, it is important to analyze and reflect on the topic. Based on this, the objective of this research was to observe the topics related to death that are included in the medical curriculum of the Brazilian universities. It is a qualitative and descriptive documentary study, executed through the observation of the syllabus, curriculum and pedagogical proposal of the 50 best medical courses of the country, according to the Folha University Ranking. The work made it possible to highlight the shortcoming in the approach to thanatology in these courses, highlighting the need to modify this reality even in the academic period, both by the structuring of academic leagues, and by the continued teaching of content and teacher training.

Keywords: Death, universities, Brazil, medical education, thanatology. 


\section{Introdução}

A morte, enquanto fenômeno transcendente e universal, tem sido expressa das mais diferentes formas ao longo da história, conforme os aspectos religiosos, culturais e sociais das populações que a vivenciam. A tanatologia, ciência dedicada ao estudo da finitude e processo de luto, tem como foco de análise a compreensão dessas relações no decorrer do tempo, bem como o papel do decesso na constituição da identidade cultural das populações (Marta, Marta, Filho, Job, 2009).

Até meados do século XIX, o ato de morrer se desenvolvia no leito domiciliar, com o doente assistido por familiares e entes queridos (Marta, Marta, Filho, Job, 2009). O médico, nesse contexto, era encarregado apenas da função de expectador, pois compreendia a morte como elemento natural e inerente à experiência existencial (Mello, Silva, 2012).

Entretanto, com o despontar do modelo biotecnicista e hospitalocêntrico no século XX, o óbito passou a ocorrer com maior frequência no ambiente hospitalar, precedido pela implementação de terapêuticas obstinadas, mesmo que, para isso, 0 bem estar e resquícios de qualidade de vida do doente fossem desconsiderados. Os médicos, entrementes, tornaram-se soldados, cujo intuito, além de combater o perecimento, era regular o momento em que ele ocorreria (Costa, Rocha, 2017).

Ao assumir essa responsabilidade, os profissionais referidos acima se defrontaram com situações nas quais não foi possível corresponder às expectativas que a sociedade e eles próprios depositaram sobre si, e frustraram-se diante da própria impotência. Dessarte, a neutralidade e indiferença mostraram-se como alternativas viáveis ao sofrimento desfrutado, tendo sido inseridas enquanto ferramentas essenciais da vivência médica (Marta, Marta, Filho, Job, 2009).

Muitos são os discursos que evidenciam o quão atual ainda é essa realidade. Em 2013, por exemplo, constatou-se que $37 \%$ dos médicos intensivistas de um hospital universitário no Rio de Janeiro acreditavam na morte como um processo triste e angustiante para o profissional da saúde, além de constantemente associado ao sentimento de culpa e receio (Almeida, Falcão, 2018). O possível motivo para tal situação, conforme apontou uma pesquisa realizada entre médicos paliativistas da Unifesp, foi a falta do ensino da tanatologia na graduação médica (Bifulco, lochida, 2009). 
Kübler-Ross, médica psiquiatra e autora da obra "Sobre a morte e o morrer", foi uma das mais importantes estudiosas a combater essa carência e ressaltou a necessidade de reflexão acerca da morte como aspecto constitucional da vida. Por meio do livro supracitado, a escritora passou a ser reconhecida como pioneira no processo da sistematização da tanatologia, definindo-a enquanto ciência dedicada ao estudo dos estágios da morte e do processo de luto (1998).

Apesar dos esforços realizados por diversos estudiosos para difusão da referida obra, é fato a vigência atual de uma doutrina ainda equivocada, segundo a qual os graduandos de medicina são distanciados do decesso e aproximados da ciência e da técnica. Assim, tornam-se incapazes de compartilhar suas angústias associadas à finitude, o que acarreta na formação de profissionais emocionalmente vulneráveis e instáveis (Marta, Marta, Filho, Job, 2009).

Essa realidade, aliás, se contrapõe às próprias competências e habilidades pregadas pelas Diretrizes Curriculares de medicina (resolução $n^{\circ} 3$, de 20 de junho de 2014), que em seu capítulo 3 , artigo 23 , coloca a compreensão do processo de morte enquanto função médica (Portal do MEC, 2018). Afinal, este é um entendimento cada vez mais requisitado pelo próprio Conselho Federal de Medicina, o qual, por meio da resolução $n^{\circ} 1995 / 2012$, regulamentou o dever de seus profissionais quanto ao entendimento das diretrizes antecipadas de vontade dos pacientes (Portal Médico, 2012).

O Código de Ética Médica (CEM), ao abordar as atribuições médicas diante de situações irreversíveis, segue essa mesma linha de raciocínio. Segundo seu capítulo 1, XXII, é preciso propiciar aos pacientes em condição terminal todos os cuidados paliativos apropriados, o que exige uma adequada capacitação para as temáticas de morte e terminalidade ainda no período acadêmico (Portal Médico, 2009).

Com base nesses fatos, evidencia-se a necessidade de analisar o estudo da morte e do morrer no conteúdo curricular da graduação médica brasileira, com vista ao desenvolvimento de reflexões para criação de estratégias que auxiliem nas dificuldades do médico relacionadas à terminalidade da vida.

\section{Objetivos}

O objetivo desta pesquisa foi investigar e analisar tópicos relacionados à morte que constam no currículo de formação médica nas universidades brasileiras. 


\section{Métodos}

Na perspectiva de elucidar as possíveis lacunas referentes ao ensino da tanatologia na graduação médica brasileira, o presente trabalho realizou uma análise curricular dos 50 melhores cursos de medicina do Brasil no ano de 2017. Trata-se de uma pesquisa com base documental e padrão qualitativo-descritivo.

Inicialmente, realizou-se a busca por um ranking confiável, que listasse as graduações médicas de maior excelência no Brasil. Por possuir parâmetros de avaliação claros e nacionalmente reconhecidos, o Ranking do Ministério da Educação (MEC) mostrou-se como opção viável, mas logo foi verificado que ele não englobava instituições de ensino superior às quais não realizavam o exame nacional de desenvolvimento (ENADE). Assim, universidades de reconhecimento internacional, como USP, não seriam analisadas.

A partir dessa problemática, passou-se a buscar outra classificação a qual mantivesse as características já citadas. Ao englobar os critérios de qualidade do ensino, educadores com mestrado e doutorado, avaliação do mercado, nota do ENADE, professores com dedicação integral e parcial, e avaliação dos docentes, o Ranking Universitário Folha (RUF) correspondeu às expectativas propostas, e foi, portanto, selecionado para a presente pesquisa (Folha de São Paulo, 2017).

Os critérios de inclusão para este estudo foram os seguintes: graduações de medicina compreendidas entre o ranking das 50 melhores do país conforme o RUF, cujos currículos, propostas pedagógicas e ementas de suas disciplinas estivessem disponíveis nos sites das instituições. Quanto aos critérios de exclusão, foram os listados a seguir: graduações de medicina não compreendidas entre as 50 melhores do país conforme o RUF; universidades com currículos, propostas pedagógicas e/ou ementas das respectivas disciplinas indisponíveis no site da instituição.

Com o Ranking de 2017 em mãos, realizou-se a verificação do currículo e proposta pedagógica dos 50 melhores cursos de medicina brasileiros, na busca por disciplinas, fossem elas obrigatórias, optativas ou eletivas, que contemplassem os diversos aspectos da tanatologia. A pesquisa englobou apenas a leitura das ementas de cada disciplina disponíveis de modo online pela respectiva instituição, mas não observou os tópicos específicos abordados em cada aula.

Deve-se ressaltar que não foram englobados projetos de extensão, ligas acadêmicas, ou quaisquer outras atividades relacionadas à morte e cuidados de fim de 
vida. Quanto aos temas de aula ministrados em cada disciplina e observados no estudo, foram os seguintes: diretrizes antecipadas de vontade; conceitos de eutanásia, distanásia, mistanásia e ortotanásia; comunicação de más notícias; impacto do envelhecimento e a perspectiva da morte; suicídio assistido; lugar da perda e da morte na experiência humana; estudo da morte e seus fenômenos; cronologia da morte; reflexões sobre vida, fé e morte na medicina; e terminalidade.

A partir disso, as graduações foram divididas conforme o modo de abordagem da tanatologia nos grupos A, B, C e D, os quais, por sua vez, foram definidos abaixo.

$\checkmark$ Grupo A: graduações nas quais a tanatologia ou similar foi verificada como disciplina obrigatória;

$\checkmark$ Grupo B: Graduações em que a tanatologia ou similar foi verificada enquanto disciplina optativa ou eletiva;

$\checkmark$ Grupo C: graduações que apresentam em sua grade curricular obrigatória matérias que tangenciam o currículo da tanatologia;

$\checkmark$ Grupo D: graduações nas quais os temas relativos à tanatologia ou similar não foram observados dentre as ementas das disciplinas ministradas.

\section{Resultados}

A análise dos 50 melhores cursos de medicina brasileiros segundo o Ranking da Folha (RUF) e com foco no estudo da tanatologia permitiu constatar os resultados dispostos na tabela abaixo.

Tabela 1: Abordagem da tanatologia nas 50 melhores graduações em medicina do Brasil, conforme o RUF

\begin{tabular}{|c|c|c|}
\hline Classificação & $\begin{array}{c}\mathbf{N}^{\circ} \text { absoluto de Universidades } \\
\text { por grupo }\end{array}$ & $\begin{array}{c}\text { Percentual em relação à } \\
\text { amostra total }\end{array}$ \\
\hline Grupo A & 2 & $4 \%$ \\
\hline Grupo B & 9 & $18 \%$ \\
\hline Grupo C & 16 & $32 \%$ \\
\hline Grupo D & 23 & $46 \%$ \\
\hline
\end{tabular}

Fonte: Elaborada pelo autor, 2018. 
Conforme se verifica na Tabela 1, das 50 universidades investigadas, somente $2(4 \%)$ compõem o grupo A, no qual a disciplina é oferecida enquanto obrigatória para o currículo; 23 universidades (46\%) compõe o grupo $D$, em que a temática não é trabalhada durante a formação acadêmica. Quanto às instituições que oferecem a matéria como optativa ou eletiva (grupo B), correspondem a $18 \%$ do total de faculdades averiguadas ( 9 graduações). Os $32 \%$ restantes (16 universidades), referentes ao grupo $\mathrm{C}$, dizem respeito àquelas que discutem o tema de modo tangencial, conforme a observação das ementas das disciplinas.

\section{Discussão}

Ao verificar a somatória percentual entre os grupos C e D, temos que $78 \%$ de nossa amostra total de universidades praticamente não abordam assuntos relativos à tanatologia. Na verdade, deve-se considerar que desde os ensinos fundamental e médio o debate em torno do assunto deixa a desejar, o que acarreta num impacto emocional ainda mais significativo para o futuro médico (Incontri, Santos, 2011).

No ambiente universitário, o distanciamento em relação à morte pode ter início já nas aulas de anatomia topográfica, em cuja figura do cadáver é apresentada somente como objeto de estudo, de modo a ignorar o ser histórico e biopsicossocial que existia naquele corpo. Mais tarde, o afastamento relativo ali criado se agrava a partir do convívio com o doente moribundo e é facilitado pelas diversas tecnologias utilizadas no referido meio (Figueiredo, Stano, 2013).

A graduação, portanto, estimula o estudante a olhar de modo imparcial para o paciente, desviando-o das relações de vínculo e aproximando-o do tecnicismo. Nessa perspectiva, o discente é desencorajado a desenvolver habilidades relacionadas às situações de terminalidade encontradas e essas últimas nem ao menos são reconhecidas como competências da profissão (Azeredo, Rocha, Carvalho, 2011).

No Brasil, esses ideais tecnicistas foram introduzidos por meio da reforma universitária de 1968, quando da imposição do modelo flexneriano. Dessa forma, houve um excesso de valorização da técnica em detrimento das ciências humanas e da ética, o que acarretou no comprometimento da visão integral ao paciente. Para piorar, a essência desse modelo de ensino transpassou os muros das universidades, ganhando forma, inclusive, na educação básica (Camargo, Almeida, Morita, 2014; Incontri, Santos, 2011). 
Haja vista a discussão em torno da morte ter sido tanto deficitária no ensino básico quanto no superior, o médico passou a ser visto em situação similar ao resto da população, inserido em uma sociedade na qual o decesso é considerado tabu (Incontri, Santos, 2011). Desse modo, a despeito das próprias diretrizes curriculares de medicina, são formados indivíduos incapazes de lidar com o morrer apropriadamente, e que cultivam os sentimentos de negação e racionalização quando expostos a esse fenômeno (Santos, Aoki, Oliveira-cardoso, 2013).

Diante disso, é clara importância da introdução de movimentos como a medicina preventiva e comunitária, que incentiva a formação de médicos generalistas mais humanos e críticos. Sua aplicação permite a formação de cidadãos pautados nas concepções estabelecidas pelo Código de Ética Médica e pela bioética, na perspectiva de desenvolver uma assistência integrada e baseada nos princípios da cidadania. Para a concretização de tal tarefa, é essencial que se repense acerca do modelo de ensino, de modo a desconstruir a concepção de ética enquanto fundamento teórico e pouco prático (Camargo, Almeida, Morita, 2014).

Isso se justifica porque, na vivência médica, há uma obstinação pela logística anti-tanásica, na qual se quer a todo preço restaurar a máquina humana (Marsiglio, 2011). Como consequência, há a criação de certa tendência por parte dos médicos ao abandono dos pacientes que não correspondem às estratégias de cura, implicando em dano significativo ao paciente e sua família (Figueiredo, Stano, 2013).

Para o profissional, a busca pelo curar supera frequentemente a do cuidar e, quando essa cura não mais é possível, a frustração resultante pode ocasionar numa tríade de sintomas conhecida como síndrome de Burnout, que consiste na associação entre exaustão emocional, despersonalização e redução da realização pessoal (Masloch, Jackson, 1986; Marta, Marta, Filho, Job, 2009). Quanto ao doente, há prolongamento de sua dor e sofrimento, que caracteriza o processo de distanásia (Marsiglio, 2011).

A distanásia é uma prática impetuosamente combatida pelo preceito da não maleficência, estabelecido com o despontar da bioética principialista na década de 70 e reintroduzido pelo Código de Ética Médica em 2009 (Portal Médico, 2009). Na medicina, isso se traduz no legítimo dever de não acarretar dano intencional ao doente, através de uma restrição aos impactos indesejáveis das ações terapêuticas. (Neves, Siqueira, 2010). 
Baseado no aforismo supracitado, o Conselho Federal de Medicina (CFM) aprovou no Código de Ética Médica (CEM) de 2009 a realização da ortotanásia, que consiste em não empreender terapias inúteis e obstinadas ao doente incurável e terminal, mas sem deixar de prestar a ele todos os serviços de cuidados paliativos apropriados (Portal Médico, 2009). A despeito disso, a prática da distanásia continua a ser realizada de modo frequente no cenário prático, e constitui-se como ferramenta desmoralizadora do processo de morte.

Em 2014, a Organização Mundial da Saúde publicou um atlas global de cuidados paliativos, como forma de monitorar a preocupação com as temáticas de fim de vida e organização das redes de assistência segundo cada país. Conforme foi verificado pelo órgão, o Brasil encontrava-se com estabelecimentos isolados os quais prestam esse tipo de serviço, diferentemente de Chile e Uruguai, que já possuíam um sistema integrado de atendimento (World Health Organization, 2014).

Mais tarde, em 2015, a revista britânica The Economist Intelligence Unit divulgou uma lista que considerava a qualidade do morrer em 80 países, denominada Quality of Death Index. Atrás de nações como Mongólia $\left(28^{\circ}\right)$ e Panamá $\left(31^{\circ}\right)$, o Brasil obteve a $42^{\mathrm{a}}$ colocação no ranking, também resultado de seu déficit quanto à preocupação com a finitude e construção de mais redes de estabelecimentos especializados em cuidados paliativos. Cinco anos antes, em 2010, o Brasil encontrava-se na $38^{\mathrm{a}}$ posição de 40 países (The Economist Intelligence Unit, 2015).

Esse é o reflexo da realidade vivenciada diariamente por muitos centros de saúde brasileiros, como é o caso de um hospital universitário paranaense. Conforme verificado em uma pesquisa estruturada com o corpo médico do estabelecimento, a perda dos pacientes é um fenômeno extremamente angustiante para a equipe profissional, o que se configura como resultado da carência de espaços de discussão sobre a finitude (Morais, Viana, Camargo, Dutra, 2015).

Com base nessa premissa, a necessidade de reflexão acerca da morte deveria ser suprida ainda durante a graduação, pela criação de projetos que a contemplassem enquanto foco das discussões. Aliás, é também necessária a instituição de ambientes profissionais propícios ao compartilhamento das angústias e medos vivenciados dentro das paredes dos hospitais (Mello, Silva, 2012).

No que tange a isso, é possível citar uma atividade de extensão desenvolvida pela Federação Internacional de Associação de Estudantes de Medicina do Brasil 
(IFMSA Brazil), intitulada "Dying: a human thing". Trata-se de uma ideia concebida para ampliação das discussões sobre terminalidade no ambiente acadêmico e que conta com a participação de médicos oncologistas e paliativistas. $O$ trabalho abrange os temas de comunicação de más notícias e abordagem ao paciente em cuidados paliativos, além de contar com a partilha das vivências relacionadas à morte realizadas pelos próprios estudantes (Lacerda, Souza, Endo, Nazima, 2015).

Nos EUA, uma proposta desenvolvida para a abordagem da tanatologia foi a introdução de pacientes em cuidados paliativos nas salas de aula, com base na perspectiva de que os melhores "professores" de morte e do morrer são os indivíduos que experimentam esse fenômeno em si próprios. No país, diversos estudos demonstraram que a exposição dos profissionais da saúde aos doentes terminais é positiva, na medida em que prepara esses trabalhadores para fornecer os cuidados de fim de vida a seus pacientes (Dickinson, 2013).

Outra metodologia de ensino também utilizada nos Estados Unidos é a realização de palestras relacionadas à finitude. Apesar de eficaz, pesquisas apontaram que o método em questão carece da abordagem individual aos alunos com relação às suas próprias experiências frente a situações de terminalidade. Assim, ressaltaram a necessidade de incentivá-los a encarar seus próprios medos associados aos indivíduos em situações de morte iminente, bem como de desenvolver habilidades de comunicação antes de concluir seu processo de formação (Dickinson, 2013).

No Brasil, um estudo realizado com concluintes de medicina de uma faculdade paulista demonstrou que os alunos concordam com a necessidade de enfoque à singularidade do estudante e propõem o debate sobre a finitude a partir da discussão de casos clínicos em pequenos grupos (Camargo, Almeida, Morita, 2014). Baseado nessa perspectiva, outro trabalho apontou que as modificações da metodologia do ensino da bioética são capazes de ampliar o interesse e participação dos estudantes, a partir da introdução de filmes, dramatizações e construção de um saber com envolvimento multiprofissional (Silva, Leão, Pereira, 2013).

Como modo de complementar esse projeto, pesquisas realizadas na última década demonstraram que a realização de cursos de ensino-aprendizagem sobre a morte e o morrer é capaz de reduzir o sofrimento dos estudantes em sua prática clínica, e que sua realização por professores, de quaisquer que sejam as disciplinas do curso médico, é essencial. Isso se justifica porque, independentemente da área 
da medicina na qual o profissional atua, é praticamente impossível não ter lidar, ao menos uma vez, com pessoas as quais estão em processo de morte ou de luto (Servaty-Seib, Parikh, 2013).

Com o devido empenho, as estratégias supracitadas podem ser eficazes para a transformação do referido cenário, mas o grande obstáculo para isso está no desenvolvimento de propostas educacionais que possibilitem a introdução desses valores na prática. Afinal, em $46 \%$ dos 50 melhores cursos de medicina brasileiros a tanatologia parece ser negligenciada, e modificar essa realidade exige um esforço coletivo e bem estruturado.

Esse enfoque prático é utilizado por uma faculdade de medicina mineira, uma das poucas no país a oferecer a disciplina de tanatologia e cuidados paliativos de modo continuado. Seus alunos, ao cursarem a disciplina no segundo, quarto e sexto ano de curso, são capacitados a oferecer aos pacientes os melhores recursos de cuidado, mesmo quando a cura já não é mais possível (Morais, Viana, Camargo, Dutra, 2015).

Trata-se de uma priorização à visão holística do ser humano, a qual também inclui a morte como fase indissolúvel e cujo estudo é essencial ao ensino médico. A inserção de seus conteúdos, portanto, integra os diversos aspectos do conhecimento, com respeito à pluralidade de emoções, crenças individuais, e abrangência significativa do $1^{\circ}$ ao $12^{\circ}$ período de curso (Incontri, Santos, 2011).

Em suma, apesar do elevado grau de complexidade da situação verificada, o ensino da tanatologia pode ir de encontro à escassez de discussões sobre morte durante a graduação, e propiciar ao aluno a capacidade de perceber o ser humano como um todo. Aliás, outras disciplinas deveriam dar maior grau de importância às emoções geradas no estudante em função de sua formação, já que a terminalidade é parte integrante da grande maioria das especialidades médicas (Azeredo, Rocha, Carvalho, 2011).

Uma das possibilidades para essa proposta é a introdução precoce do estudante no ambiente de Unidade de Terapia Intensiva, realizada em disciplinas como cardiologia, nefrologia, neurologia e medicina intensiva. Como a terminalidade é também rotina nessas especialidades, as discussões em torno do tema poderiam ser aplicadas de modo prático e direcionado. $O$ decesso poderia, pois, ser apresentado enquanto etapa infracionável da vida, e também como instrumento para pensar no indivíduo além da doença (Almeida, Falcão, 2018). 
Outra intervenção também bastante resolutiva, conforme mostrou um trabaIho sobre cuidados paliativos no Brasil, é a estruturação de ligas acadêmicas que discutam sobre o processo de morte. A partir delas, é possível não só habilitar os estudantes e corpo docente a lidar com o tema, mas também fomentar a criação de estratégias e recursos para a concretização de tal tarefa. É o caso de uma liga acadêmica desenvolvida em uma universidade do Nordeste brasileiro, a qual, anualmente, promove cursos e palestras abertos ao público sobre dor e cuidados de fim de vida (Garcia, Rodrigues, Lima, 2014).

\section{Conclusão}

A análise da ementa e grade curricular dos 50 melhores graduações em medicina do Brasil permitiu constatar um déficit quanto à capacitação de seus estudantes para as discussões em torno da terminalidade. Por não se sentirem preparados, muitos dos profissionais formados ignoram a figura do paciente enquanto ser biopsicossocial, e aproximam-se institivamente da ciência e da técnica.

Diante disso, é clara a necessidade de incrementar discussões relacionadas à tanatologia em todas as graduações de medicina e o ensino teórico-prático de suas atribuições de modo continuado ao longo da faculdade. Para tal, é necessário o desenvolvimento de um trabalho de conscientização do corpo docente sobre a relevância do tema e disponibilização de capacitações que preparem o referido público para essa abordagem com os discentes. Em conjunto, é importante a concepção de disciplinas correlatas às quais contemplem direta ou indiretamente a finitude; assim como a fundação de ligas acadêmicas em que a morte seja o foco das discussões.

\section{Referências}

1. Almeida LFD, Falcão EBM. Representação Social de Morte e a Formação Médica: a Importância da UTI. Revista Brasileira de Educação Médica 2018; 37(2): 226-34.

2. Azeredo NSG, Rocha CF, Carvalho PRA. O Enfrentamento da Morte e do Morrer na Formação de Acadêmicos de Medicina. Revista brasileira de educação médica $2011 ; 35(1)$ : 37-43.

3. Bifulco VA, lochida LC. A formação na graduação dos profissionais de saúde e a educação para o cuidado de pacientes fora de recursos terapêuticos de cura. Revista Brasileira de Educação Médica 2009; 33(1): 92-100. 
4. Camargo, A, Almeida, MAS; Morita, I. Ética e bioética: o que os alunos do sexto ano médico têm a dizer. Revista Brasileira de Educação Médica 2014; 38(2): 182-9.

5. Costa ICD, Rocha ACD. Percepções da morte e do morrer para residentes de medicina em um hospital terciário. Revista Ciências em Saúde 2017; 7(4): 7-14.

6. Dickinson, G. End-of-life and palliative care curricula in U.S. social work graduate programs. Illness, Crisis \& Loss 2013; 21(4): 315-24.

7. Figueiredo MDGMCA, Stano RDCMT. O Estudo da Morte e dos Cuidados Paliativos: Ausências no Currículo de Medicina. Revista Ciências em Saúde 2013; 3(3): 74-86.

8. Figueiredo MDGMCA, Stano RDCMT. O Estudo da Morte e dos Cuidados Paliativos: uma Experiência Didática no Currículo de Medicina. Revista brasileira de educação médica 2013; 37(2): 298-307.

9. Folha de São Paulo. Ranking universitário folha 2017. Acessível em: http://ruf. folha.uol.com.br/2017/ranking-de-cursos/medicina/.

10. Garcia JBS, Rodrigues RF, Lima SF. A estruturação de um serviço de cuidados paliativos no Brasil: relato de experiência. Revista Brasileira de Anestesiologia 2014; 64(4): 286-91.

11. Incontri D, Santos FS. As leis, a educação e a morte - uma proposta pedagógica de tanatologia no Brasil. International Studies on Law and Education 2011; 9(1): 73-82. Acessível em http://www.hottopos.com/isle9/73-82Dora.pdf.

12. Kübler-ross E. Sobre a morte e o morrer. In: Kübler-ross $E$ (8 ed.). Atitudes sobre a morte e o morrer. São Paulo: Martins Fontes; 1998: 17-21.

13. Lacerda PNM, Souza LR, Endo RM, Nazima MTST. Extensão universitária na atualidade: a IFMSA Brazil como foco. Revista de Medicina 2015; 94(2): 81-6.

14. Marsiglio CF. Ensino de cuidados paliativos na graduação do curso de medicina (monografia). Florianópolis: Universidade Federal de Santa Catarina; 2011.

15. Marta GN, Marta SN, Filho ADA, Job JRPP. O estudante de Medicina e o médico recém-formado frente à morte e ao morrer. Revista brasileira de educação médica 2009; 33(3): 405-16.

16. Maslach, C, Jackson, SE. Maslach Burnout Inventory. In: Maslach, C, Jackson, SE (2 ed). Palo Alto: California; 1986.

17. Mello AAM, Silva LCD. A Estranheza do Médico Frente à Morte: Lidando com a Angústia da Condição Humana. Revista da Abordagem Gestáltica 2012; 18(1): 52-60.

18. Morais ARGD, Viana LFDS; Camargo APD; Dutra, FDO. A importância da formação paliativista no currículo médico brasileiro: quando o curar não é possível. Revista Uningá 2015; 46(1): 22-8.

19. Neves NMBC, Siqueira JED. A bioética no atual Código de Ética Médica. Revista Bioética 2010; 18(2): 439-50. 
20. Portal do MEC. Diretrizes curriculares nacionais do curso de graduação em medicina. Acessível em: http://portal.mec.gov.br/cne/arquivos/pdf/Med.pdf.

21. Portal Médico. Resolução CFM no 1.995/2012. Acessível em: http://www.portalmedico.org.br/resolucoes/cfm/2012/1995_2012.pdf.

22. Portal Médico. Código de ética médica - resolução CFM n 1931/09. Acessível em: https://portal.cfm.org.br/images/stories/biblioteca/codigo $\% 20$ de $\% 20$ etica $\% 20$ medica.pdf.

23. Santos MAD, Aoki FCDOS; Oliveira-Cardoso, EAD. Significado da morte para médicos frente à situação de terminalidade de pacientes submetidos ao Transplante de Medula Óssea. Ciência e saúde coletiva 2013; 18(9): 2625-34.

24. Servaty-Seib, HL, Parikh, SJT. Using service-learning to integrate death education into counselor preparation. Death Studies 2013; 38(3): 194-202.

25. Silva J, Leão MHM, Pereira ACAC. Ensino de bioética na graduação de medicina: relato de experiência. Revista Bioética 2013; 21(2):338-43.

26. The economist intelligence unit. The 2015 Quality of Death Index. The economist intelligence unit limited 2015; 71.

27. WHO. World Health Organization. Global atlas of palliative care at the end of life. Londres: WHO; 2014.

Recebido: 13/03/2019. Aprovado: 17/07/2019. 